\title{
Automated System for Determining Frequency Dependent Parameter Model of Transmission Line in a Laboratory Environment
}

\author{
Aaron St. Leger*, Valentina Cecchi ${ }^{+}$, Megha Basu, Karen Miü, and Chika Nwankpa ${ }^{\#}$ \\ * Department of Electrical Engineering and Computer Science, United States Military Academy, West Point, NY USA \\ +Department of Electrical and Computer Engineering, The University of North Carolina, Charlotte, NC USA \\ \# Electrical and Computer Engineering Department, Drexel University, Philadelphia, PA USA
}

Abstract--This work presents an automated system for determining transmission line model parameters at various frequencies. The system was developed and implemented on General Electric test reactors designed to model power transmission line behavior in a laboratory environment. The measurement system and data processing are controlled and automated via custom developed LabVIEW software. Measurements are obtained from voltage and current probes interfacing with oscilloscopes. This data is then acquired and processed in LabVIEW software. The structure of the transmission line model, desired confidence level and number of tests to conduct are specified user inputs. The system subsequently provides the transmission line impedance parameters based on these specifications. These parameters are calculated using both time domain and frequency domain techniques. While this work focuses on determining transmission line model parameters, this automated test measurement system is applicable to any device that can be parameterized via a current-voltage (I-V) characteristic or frequency response.

Keywords: Impedance measurement, Uncertainty, Digital signal processing, RMS measurement, Frequency dependence, Experimental Analysis

\section{Introduction}

Power systems exhibit both fundamental and non-fundamental frequency components [1]. An emerging characteristic of modern power systems is an increased level of non-fundamental frequency components attributed to the augmented use of power electronic switches. Common power electronic devices introduce a wide range of harmonics [2]. Specifically, up to the $39^{\text {th }}$ harmonic should be monitored in accordance with power quality standard EN50160 [3]. In addition, up to the $15^{\text {th }}$ harmonic component is introduced into the network in a capacitor switching scenario. Under the increasing presence of non-fundamental frequency components, it is necessary to characterize components at various frequencies and develop appropriate models.

State of the art simulation tools utilize manufacturer specifications. However, these specifications are often made at rated conditions (e.g. $50-60 \mathrm{~Hz}$ in power systems). It is often the case that operating conditions seen by hardware are not at rated levels and electrical parameters can vary based on current levels, voltage levels and frequency. In order to obtain accurate simulation results, and subsequently proper system control strategies, these parameters must be known for various operating conditions. The automated test measurement system developed in this work can determine parameters at different voltages, currents, and frequencies.

* Corresponding author.

E-mail addresses: aaron.stleger@usma.edu (A. St. Leger), vcecchi@uncc.edu (V.Cecchi),karen@ece.drexel.edu (K. Miu), con22@drexel.edu (C. Nwankpa) 
Specifically, to account for non-fundamental frequencies in power systems, it is desirable to know electrical network parameters at different frequencies. Generally, non-fundamental frequencies are undesirable in power systems. Therefore, it is difficult to test for parameters at non-fundamental frequencies within the power system itself. Thus, an automated test system for determining frequency dependent parameters is desirable. The proposed automated test measurement system presented here facilitates experimental trials on power system components. The focus is on tests at various frequencies and determining parameters for each frequency tested. This system was developed in conjunction with a method for modeling transmission lines at non-fundamental frequencies [4] which incorporates frequency dependent parametric variations such as skin effect.

Frequency dependency is accounted for in this modeling approach by developing line models with a finite number of segments. As such, the transmission line representation in this work consists of a finite number of segments. In addition, statistical analysis techniques are implemented when determining model parameters similar to the approaches discussed in [5]. The system presented here can easily be extended to other techniques such as frequency response analysis on power transformers [6] or developing frequency dependent models of other components.

Statistical techniques allow for evaluation of confidence levels and confidence intervals of the transmission line model parameters. Some examples applied to impedance measurements can be seen in $[5,7]$ Many trials are necessary in order to increase confidence in results. Thus, automation facilitates the experimental trials. In addition, the proposed automation results in a substantial savings in time and requires no user interface other than hardware set up and software initialization. Similar work on test automation has been performed to model high power automotive batteries [8], determine magnetization characteristics of switched reluctance motors [9] and automate analysis of periodic signals [10]. In this case, the impedances of a hardware test setup for power transmission line models are determined. This work incorporates some measurement principles of prior work and expands upon it by incorporating statistical analysis into the testing process.

Applications of an automated test measurement system for transmission line model parameters are now discussed. It should be noted that the hardware under test in this work is not an actual transmission line, but hardware that was specifically designed can constructed for the purposes of emulating and studying power transmission line behavior in the laboratory environment. The main contribution of this work is the development of a systematic process of accurately defining the transmission line parameters and automation of this process. If these hardware components are accurately defined, a robust virtual laboratory could be developed as shown in [11]. A method for handling parameter uncertainty in simulation is presented in [12]. However, in order to utilize such approaches a detailed model of parameters and their uncertainty is required. Specifically, in power system operation the uncertainty of transmission line parameters has a direct effect on the power transfer capability of the system [13]. With a statistically based model of transmission line parameters, the power transfer capability and limits of the system can be more clearly defined and understood. Moreover, the systematic process presented in this paper is generalized in such a way to be applied to any device that can be parameterized via a current-voltage (I-V) characteristic or frequency response.

The paper is organized as follows. The next section provides an overview of the test and measurement system setup. The following section details the testing procedure and statistical analysis. Then, the software development is shown. Experimental results obtained from a three segment transmission line are then presented. The hardware experiments were performed within the power systems laboratories at Drexel University [14]. 


\section{Hardware Setup}

The hardware setup of the automated test measurement system consists of power and measurement components. The power components consist of a power source and the circuit to be tested. Current probes, voltage probes, and oscilloscopes are employed for measurement. The configuration and acquisition of data from this hardware is controlled through the LabVIEW software. Additionally, this software handles the processing of the data and determination of transmission line parameters.

\subsection{Power Hardware}

A hardware setup was designed to test different transmission line models. The transmission line models to be tested can be changed in structure ( $\Gamma$ or $\pi[15])$ and the number of segments. This particular modeling methodology is valid for a per-phase analysis of a three phase line operating under balanced conditions. The power hardware setup for a one segment gamma $(\Gamma)$ transmission line is shown in Fig. 1. The hardware setup consists of:

- A controllable source (Kikusui PCR-6000W2 [16]), which enables a frequency variation from 0 to $500 \mathrm{~Hz}$ and voltage output from 0 to $300 \mathrm{~V}$ RMS.

- A transmission line box, which consists of numerous General Electric (GE) portable test reactors (Part \#: 6054975 G1) [17] and capacitors (GE A28F5602).

- A variable resistive load consisting of a rheostat.

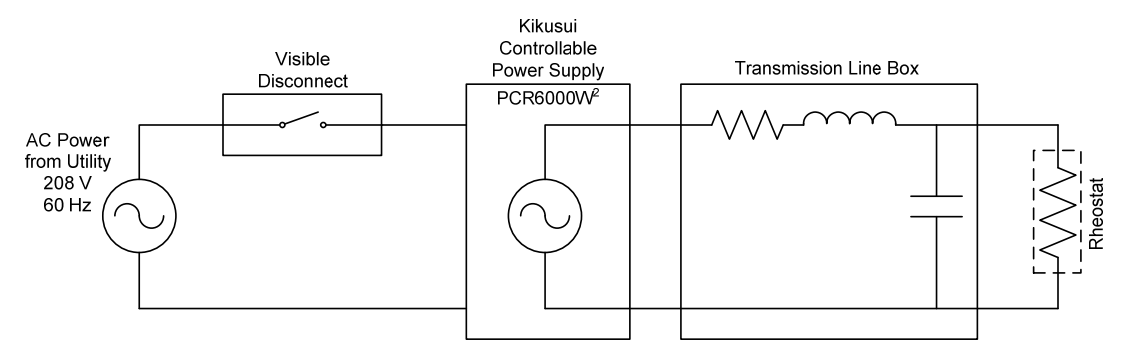

Fig. 1. Power Hardware Test Setup for One Segment Gamma Transmission Line Model

The hardware setup described is capable of testing frequencies up to $500 \mathrm{~Hz}$ for linear loads. Two methods may be employed to test higher frequencies. First, a source capable of outputting higher frequencies may be used. Second, non-linear loads inducing higher frequency harmonics may be interconnected. If non-linear loads are used then voltage and current magnitudes can be determined directly from FFT. Impedance parameters can then be determined at each frequency of interest.

\subsection{Measurement Hardware}

The measurement hardware setup consists of several measurement devices connected to the power hardware. In particular, it allows for voltage and current waveform capture at up to four locations. For example, the setup for a three segment gamma $(\Gamma)$ transmission line is shown in Fig. 2 and includes voltage and current measurements before and after each transmission line segment. 


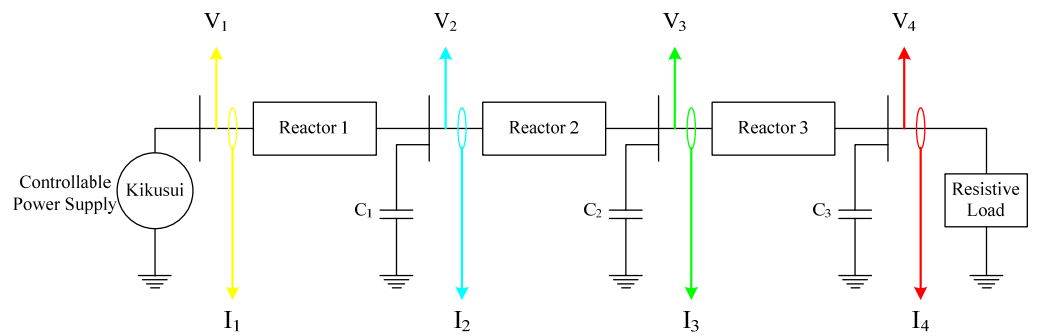

Fig. 2. Setup of a $3 \Gamma$ Segment Model

Measurement equipment records voltage and current waveforms via voltage probes (Tektronix P5200) and current probes (Yokogawa 701933) and two four-channel oscilloscopes (Tektronix 3014B). In addition, oscilloscopes are externally triggered synchronously through a function generator (Agilent 33220A) in order to simultaneously record up to eight waveforms.

Oscilloscope configuration, including window size and subsequently sampling rates, is handled automatically in software for each measurement. The measurement hardware setup for the three segment transmission line is shown in Fig. 3.

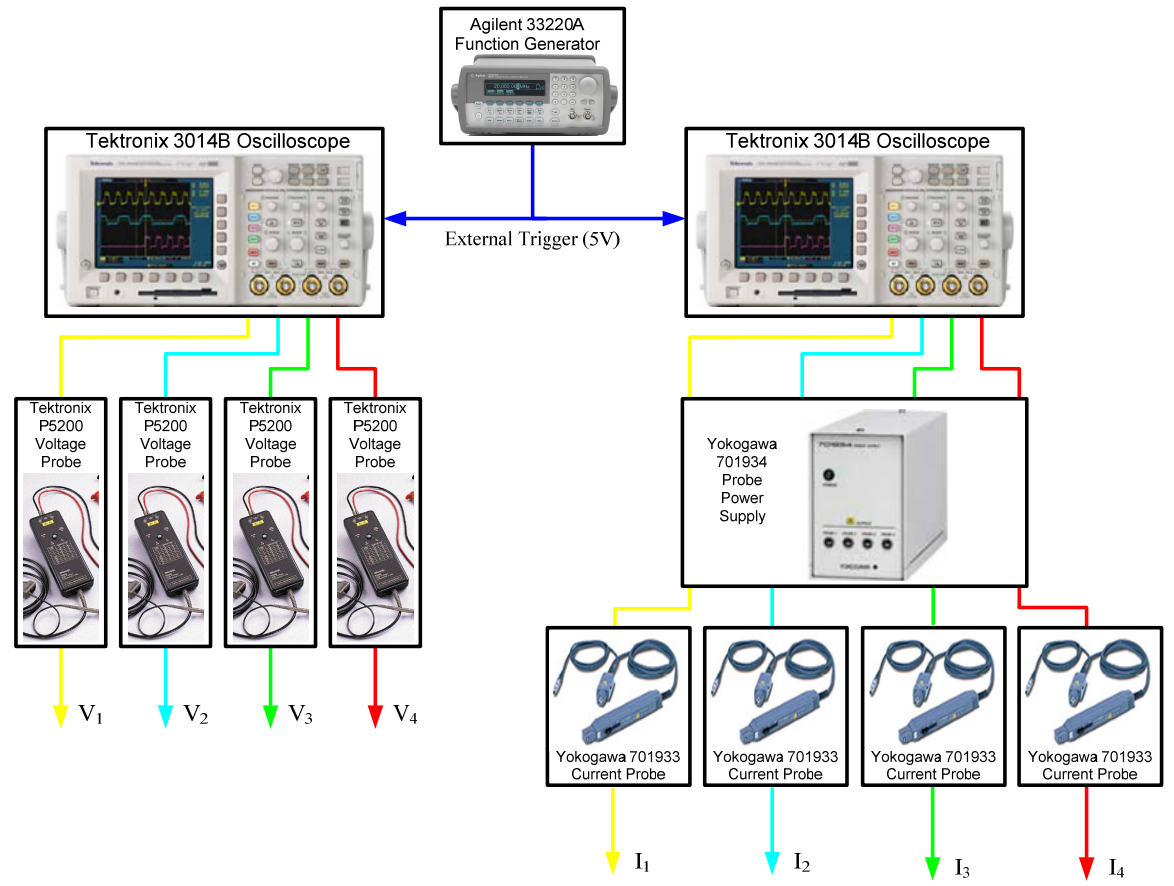

Fig. 3. Measurement Hardware Setup

From [18-20], the measurement hardware utilized has the following accuracy characteristics:

- Digital Oscilloscope Tektronix 3014B:

Vertical Accuracy $= \pm 2 \%$

Horizontal Accuracy $= \pm 0.002 \%$ per sec/div

- Voltage Probe Tektronix P5200: Accuracy $= \pm 3 \%$

- Current Probe Yokogawa 701933: Accuracy $= \pm 2 \%$

A measurement, $x_{i}$, contains components of the true value being measured, $x$, and the measurement error $v_{i}$ :

$$
x_{i}=x+v_{i}
$$


The worst case multiplicative measurement error, normalized to the true value, is determined to be:

$\pm \frac{\left(x_{i \max }-x\right)}{x}= \pm \frac{(x \cdot 1.03 \cdot 1.02-x)}{x} \%= \pm 5.06 \%$ for voltages, and $\pm \frac{(x \cdot 1.02 \cdot 1.02-x)}{x} \%= \pm 4.04 \%$ for currents.

The propagated multiplicative measurement error through the probes and scope, normalized to the true value, are determined to be:

$\pm \sqrt{\left(v_{v p}\right)^{2}+\left(v_{s c}\right)^{2}} \times 100 \%= \pm \sqrt{(.03)^{2}+(.02)^{2}} \times 100 \%= \pm 3.6 \%$ for voltages where $v_{v p}$ and $v_{s c}$ are the voltage probe and oscilloscope \% error magnitude respectively, and $\pm \sqrt{\left(v_{i p}\right)^{2}+\left(v_{s c}\right)^{2}} \times 100 \%= \pm \sqrt{(.02)^{2}+(.02)^{2}} \times 100 \%= \pm 2.8 \%$ for currents where $v_{i p}$ is the current probe \% error magnitude.

The current probes are rated for 30 amps with a bandwidth of zero (Direct Current) to $50 \mathrm{MHz}$. The voltage probes can measure up to 1000 volts from zero to $25 \mathrm{MHz}$. The voltage and current transducers were chosen based on current and voltage levels present in the power hardware. Different transducers will be required for different ranges in voltage, current, or frequency. The test procedure for determining the parameters of the transmission line model is outlined in the next section. The hardware setup is not altered during this test procedure.

\section{Test and Data Analysis Procedure}

The test procedure consists of conducting the experiment multiple times while varying the frequency and/or voltage levels. Raw waveforms of voltages and currents are acquired and processed for each experimental run. One experimental run is defined as performing all tests specified, for example, testing the hardware for all desired voltage levels and frequencies. In addition, automated statistical analysis is performed on the results. Through the implementation of statistical techniques, parameters and their associated tolerances can be determined based on the specified confidence interval(s) and number of runs.

While this process can be applied to any hardware that can be parameterized via current-voltage characteristics, the hardware tested in this work was a representation of a transmission line. The purpose was to determine frequency dependent transmission line model parameters. For this particular application, the outputs of the test procedure are the transmission line parameters as a function of frequency and the intervals of voltages, currents and impedances at each frequency tested.

A flow chart detailing the test procedure is shown in Fig. 4. It should be noted that the tasks in this figure are automatically performed by the LabVIEW software based on initial user configuration. This automation includes configuration of measurement hardware, acquisition of raw waveform data from the oscilloscopes, processing of raw waveform data, and presentation of results. The procedure can be executed once the hardware and measurement equipment have been setup and test parameters have been provided by the user. Tests execute automatically until finished, more specifically, when the specified number of runs has been performed. Input parameters required from the user are as follows:

1. Structure of the hardware under test (e.g. gamma, pi, etc.)

2. Voltage level(s) to be tested

3. Range of frequencies to be used

4. Parameters to be determined

5. Confidence level(s) of measurements or parameters 


\section{Number of runs}

The specification of these items is a prerequisite to the automated testing and processing of data. The voltage levels and range of frequencies used are required for automatic configuration of the $\mathrm{AC}$ source. In addition, these values are used for post processing and control of data acquisition equipment. Post processing also requires the user to specify the parameters to be determined, number of runs that are performed and confidence intervals. These are necessary for computing standard deviations and confidence intervals of parameters.

\subsection{Measurement and Data Processing}

The transmission line model parameters are determined based on measurement and data processing. Raw waveform data of voltages and currents are acquired by the software from the oscilloscopes. The LabVIEW software then performs all processing of the raw data and impedances are calculated from this processed data. More specifically, RMS values of currents and voltages are determined via waveform analysis (time-domain), phase determined via FFT (Frequency Domain), and impedance calculated based on the ratio of voltage and current. The following assumptions were made for this process:

1. Power source provides a pure sinusoidal waveform at a known frequency;

2. Sampling frequency is known (set by the oscilloscope);

3. Effects of probe input impedances are negligible (Impedance measured $<<$ Probe impedance);

4. Non-coherent sampling;

5. Systematic errors of the measurement system are time invariant and can be characterized and removed via calibration.

Software has been designed and implemented to process this data and to compute the impedance parameters of the transmission line model. These automated functions include:

- Oscilloscope

oVertical scaling

oHorizontal scaling (dictates sampling rate)

o Triggering/Data Acquisition

- Waveform

oComputing waveform average (DC offset)

oComputing RMS values

oComputing phase angles

oComputing Impedances

The measurement process accounts for changing voltage levels, current levels and frequencies during testing. Oscilloscopes were utilized to capture and acquire raw waveform data. Thus, the horizontal and vertical axes are automatically adjusted via software during testing. 


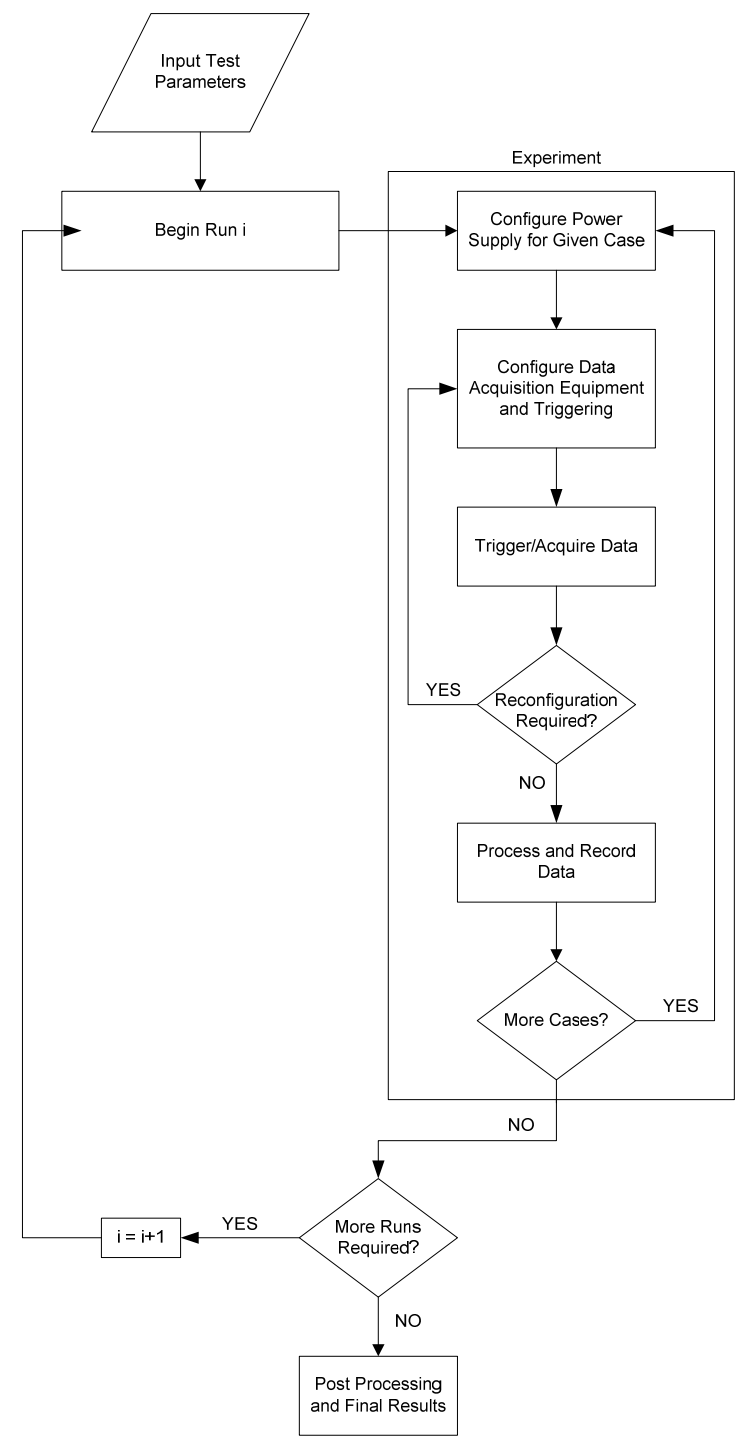

Fig. 4. Test Process Flow Chart

The horizontal axis, time, is adjusted based on the frequency under test. The appropriate horizontal scale was determined a priori to ensure fidelity in the waveform measurement. Analysis of multiple cycles is necessary when non-coherent sampling occurs, that is, when the length of the observation interval does not equal the period of the waveform being examined. This occurs on most frequencies under test due to a non-integer ratio of the sampling rate of the oscilloscope and test frequency. A windowing technique could be utilized to mitigate errors due to non-coherent sampling. However, in this work, accurate results are obtained by analyzing multiple cycles of data and averaging the results. It has been shown that measurement bias due to non-coherent measurements decreases as the number of cycles measured increases [21] and the long term average of these values converges to the true values [22]. Other research has shown that at least five cycles is recommended for sine-fitting techniques [23]. Experimentation has shown that obtaining a minimum of eight cycles exhibited convergence for the system presented here. The horizontal axis was scaled based on a lookup table which is shown in Table I. Scaling according to this table ensured that a minimum of eight cycles and a maximum of twenty cycles are obtained for all frequencies. 
Table I. Horizontal Scaling for Measurement Equipment

\begin{tabular}{|c|c|}
\hline Frequency (Hz) & Horizontal Scale (ms/div) \\
\hline 1 & 1000 \\
$2 . .3$ & 400 \\
$4 . .7$ & 200 \\
$8 . .19$ & 100 \\
$20 . .39$ & 40 \\
$40 . .79$ & 20 \\
$80 . .199$ & 10 \\
$200 . .399$ & 4 \\
$400 . .500$ & 2 \\
\hline
\end{tabular}

The adjustment of the oscilloscope vertical axis, corresponding to electrical parameters, was also automated. However, the vertical scale automation could not be done a priori. The voltage and current levels are not known explicitly before testing. These values are monitored and fed back into the software during testing. The software adjusts the vertical axes as necessary. Inappropriate scaling of the vertical axis can lead to measurement errors. Clipping of signals can occur for too small a vertical axis and poor signal to noise ratio for too large a vertical axis. An algorithm was developed and implemented to check and adjust the vertical axis on the oscilloscopes for each waveform measurement. This process is shown in Fig. 5.

The vertical axis is increased when signal clipping is detected. Clipping is detected by comparing the peak values of the signals to the peak value of the vertical axis. Conversely, the vertical axis is decreased in the presence of small signals. If the vertical axis can be scaled down to the next setting, and the resulting peak value of the signal does not result in clipping, then the signal is defined as small. If the vertical axis requires adjustment, then data is reacquired and checked once more for clipping and small signals. Data is processed after the vertical scaling is performed.

The processing of data consists of analyzing raw waveform data and computing impedance values. Each measured waveform, for a linear load, can be represented by:

$$
x(t)=A_{0}+A \cos (2 \pi f t+\phi)
$$

which is evaluated in software by estimating the waveform amplitude, $A$, phase, $\phi$, and DC component $A_{0}$. This is similar to the three parameter technique in [23]. For non-linear loads the waveform can be represented by a summation of all harmonic components and the DC component:

$$
x(t)=A_{0}+\sum_{n=i}^{N} A_{n} \cos \left(2 \pi n f t+\phi_{n}\right)
$$

where $f$ is the fundamental frequency.

Waveform processing is dependent on the number of data points per cycle of raw waveform data. The number of data points per cycle is determined by the sampling rate of the data acquisition equipment and the frequency under test: 


$$
n_{\text {cycle }}=\left\lfloor\frac{\text { sampling rate }}{f_{\text {test }}}\right\rfloor
$$

where $n_{\text {cycle }}$ is an integer of data points per cycle and $f_{\text {test }}$ is the test frequency. The rounding operator ensures an integer value.

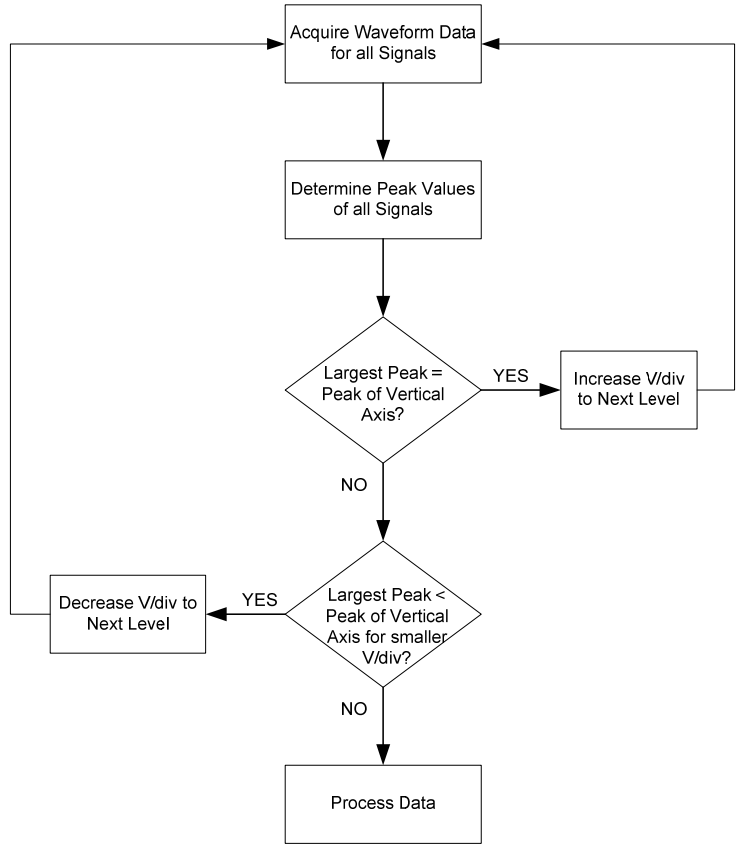

Fig. 5. Vertical Scaling Algorithm for Measurement Equipment

The raw data is first processed to evaluate if a DC offset is present in measurement. It is assumed that the source is pure AC with no offset. Thus, any offsets present in the data would be due to measurement devices and are removed from the raw data. The offset is evaluated by computing the cycle average of each waveform:

$$
A v g_{\text {cycle }}=\frac{1}{n_{\text {cycle }}} \sum_{i \in c y c l e} \text { waveform }[i]
$$

where $i$ indicates a sample that falls within a single period and $n_{\text {cycle }}$ is an integer representing the number of samples per period. The samples per period are determined based on the sample rate and frequency of the waveform.

The next step in data processing determines the RMS value and phase of each waveform from the offset corrected data. The RMS value is determined by analyzing the cycle RMS:

$$
R M S_{\text {cycle }}=\sqrt{\frac{1}{n_{\text {cycle }}} \sum_{i \in c y c l e}\left(\text { waveform }[i]-A v g_{\text {cycle }}\right)^{2}}
$$

The phase of each waveform is determined by conducting a Fast Fourier Transform (FFT) on each waveform with a Hanning window. The phase is taken at the frequency being tested and referenced to the source voltage waveform. An FFT approach was required as time domain analysis proved to be inadequate in application. Relatively small levels of uncertainty in timing when 
estimating peak or zero crossing time of waveforms resulted in a correspondingly large variation of calculated phase angles.

Once the phase angles are determined via FFT, the transmission line model parameters are then computed with the processed waveform data. Specifically, the impedance of component $i$ within a gamma structure is computed by:

$$
\left|Z_{i}\right| \angle \theta_{Z_{i}}=\frac{\left|V_{i}\right| \angle \theta_{V_{i}}}{\left|I_{i}\right| \angle \theta_{I_{i}}}=\left|Z_{i}\right| \cos \left(\theta_{Z_{i}}\right)+j\left|Z_{i}\right| \sin \left(\theta_{Z_{i}}\right)=R+j X
$$

where $\left|Z_{i}\right|$ is the impedance magnitude, $\left|V_{i}\right|$ is the voltage magnitude across the impedance and $\left|I_{i}\right|$ is magnitude of the current flowing through the impedance. $\theta_{Z_{i}}$ is the phase of the impedance, $\theta_{V_{i}}$ is the phase of the voltage across the impedance, and $\theta_{I_{i}}$ is the phase of the current flowing through impedance. The voltage and current phases are referenced to the AC source voltage. The source voltage angle is defined as zero degrees. Note that for different structure (e.g. a $\pi$ structure) this formulation would require modification.

\subsection{Statistical Analysis}

There is inherently uncertainty and error associated with the data acquisition equipment and subsequent propagation of error in the impedance calculation. Propagation of error for a function of multiple variables, $Z=f(X, Y)$ can be quantified by [24]:

$$
s_{z}=\sqrt{\left(\frac{\partial Z}{\partial X}\right)^{2} \cdot s_{X}^{2}+\left(\frac{\partial Z}{\partial Y}\right)^{2} \cdot s_{Y}^{2}\left(\frac{\partial Z}{\partial X}\right)\left(\frac{\partial Z}{\partial Y}\right) \cdot s_{X Y}^{2}}
$$

where $s_{X}, s_{Y}$, and $s_{Z}$ are the standard deviations of $\mathrm{X}, \mathrm{Y}, \mathrm{Z}$ respectively and $s_{X Y}$ the covariance between $\mathrm{X}$ and $\mathrm{Y}$ measurements. Applying this to (7), and neglecting the covariance term due to independent measurements of voltage and current, yields the following standard deviation for impedance magnitude:

$$
S_{|Z|}=\sqrt{\left(\frac{1}{I}\right)^{2} \cdot s_{V}^{2}+\left(\frac{-V}{I^{2}}\right)^{2} \cdot s_{I}^{2}}=|Z| \sqrt{\left(\frac{s_{V}}{V}\right)^{2}+\left(\frac{s_{I}}{I}\right)^{2}}
$$

where $s_{|Z|}, s_{V}$, and $s_{I}$ are the standard deviations of the impedance magnitude calculation, voltage measurement and current measurement respectively. The standard deviations of the resistance, $\left(s_{R}\right)$, and reactance, $\left(s_{X}\right)$, calculations are:

$$
\begin{aligned}
& s_{R}=\sqrt{\left(s_{|Z|} \cdot \cos \theta_{Z}\right)^{2}+\left(-s_{\theta_{z}} \cdot|Z| \sin \theta_{Z}\right)^{2}} \\
& s_{X}=\sqrt{\left(s_{|Z|} \cdot \sin \theta_{Z}\right)^{2}+\left(s_{\theta_{z}} \cdot|Z| \cos \theta_{Z}\right)^{2}}
\end{aligned}
$$

where $s_{\theta_{z}}$ is the standard deviation of the impedance angle.

Guidelines for quantifying and handling uncertainty in measurement developed in the Guide to Expression of Uncertainty in Measurement (GUM) ISO standard [25] and summarized in NIST Technical Note 1297 [26] have been applied in this paper. Type A evaluations of uncertainties are evaluated and quantified through analysis of repeated measurements. This is the 
approach used in this work. Standard deviations, confidence levels and intervals are then determined based on the number of runs and distribution of the data. It is assumed that cumulative effects result in a student-t distribution function. The standard deviation was estimated from the data as follows [27]:

$$
s=\sqrt{\frac{\sum_{i-1}^{n}\left(x_{i}-\bar{x}\right)^{2}}{n-1}}
$$

where $\bar{x}$ is the computed mean, $x_{i}$ is sample $i$, and $n$ is the number samples, more specifically, the number of calculated values obtained from measurement. For example, one sample is obtained by performing one run and computing the impedance using (7). The standard deviation is estimated for all measurements and calculated parameters. The intervals based on student-t distribution are computed as follows [27]:

$$
\bar{x}-t_{1-\frac{\delta}{2}}(n) \frac{s}{\sqrt{n}} \leq \eta \leq \bar{x}+t_{1-\frac{\delta}{2}}(n) \frac{s}{\sqrt{n}}
$$

where $\delta$ is the confidence level, $\eta$ is the mean of a random variable. The processing of data and parameter computation was all handled in software.

\section{Software}

Software was developed to control the Kikusui power supply and to acquire and process data. The software was developed using National Instruments LabVIEW [28]. The source and measurement equipment is controlled via a General Purpose Interface Bus (GPIB) based on the IEEE-488.1-2003 standard. The software interface allows the user to configure the software and hardware, specify where data from the experiment is to be saved, as well as display waveforms and processed data. Fig. 6 shows a screen capture of the voltage measurement and processing screen. Here the waveforms and processed data are displayed during testing. The raw waveforms are displayed on the screen for up to four voltages. The processed data displayed consists of voltage offset, RMS value, and phase angle of each waveform. In addition, the measurement equipment can be configured via this screen. There is a similar screen for current measurement and processing.

The transmission line model parameters are also displayed through the software. Fig. 7 shows a screen capture that displays the parameters based on processed data. 


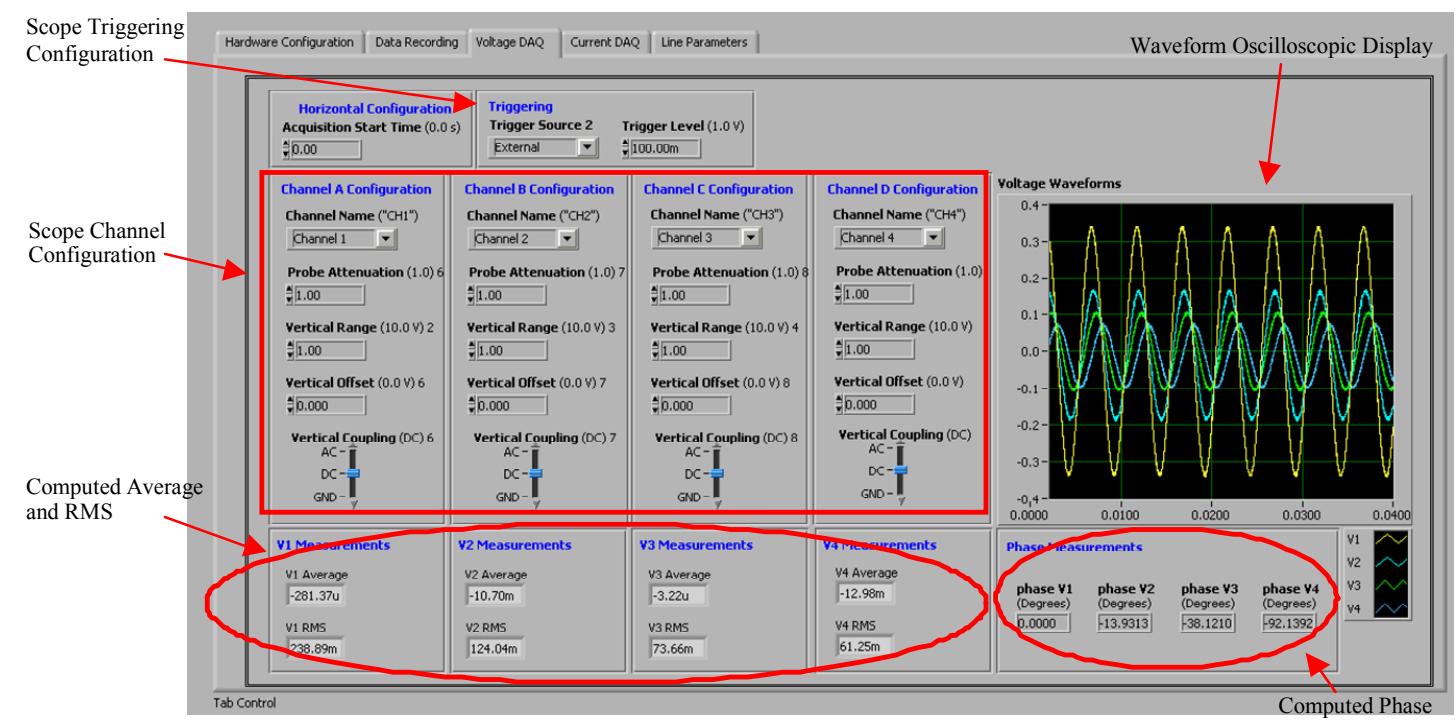

Fig. 6. Screen Capture for Voltage Measurement and Processing from Automated Test Measurement System

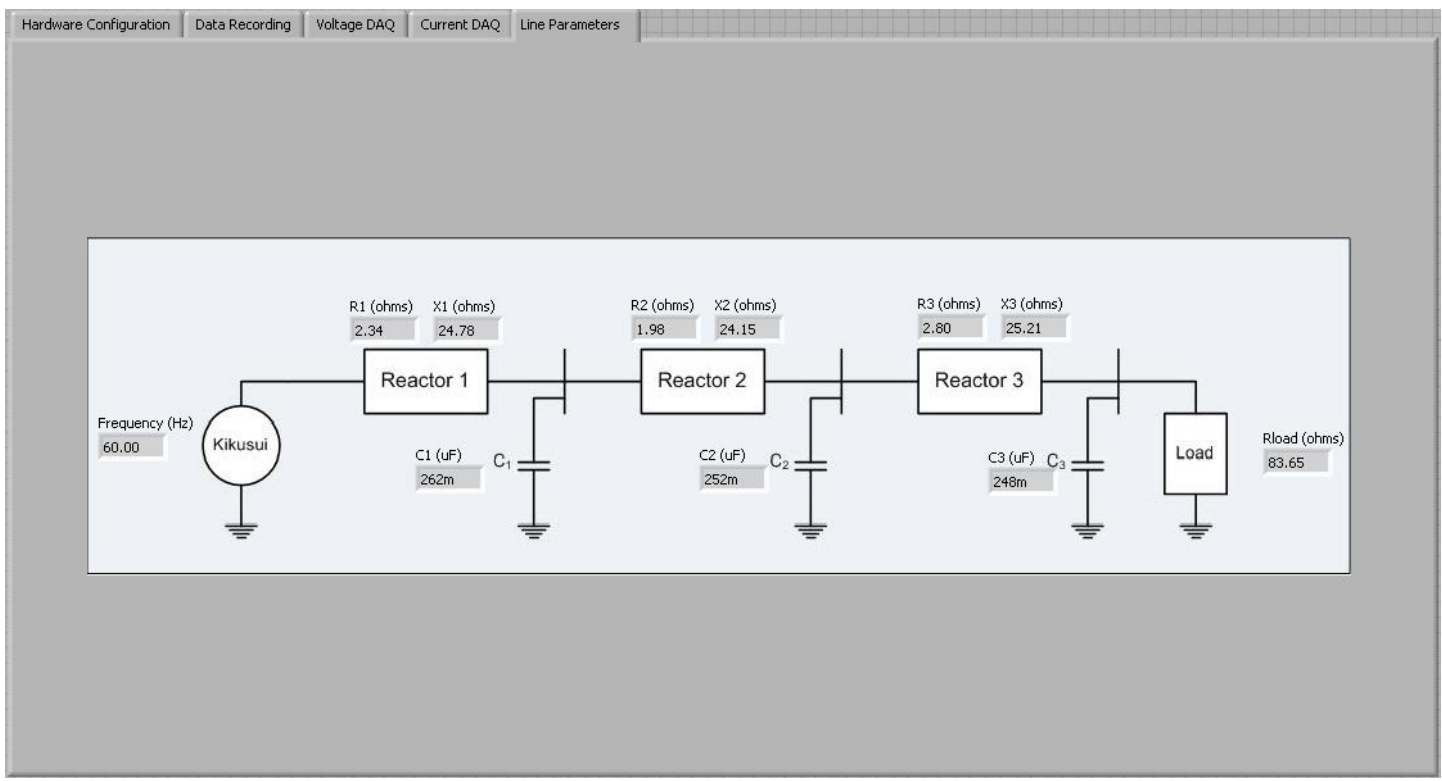

Fig. 7. Screen Capture of Transmission Line Parameters Computed from Automated Test Measurement System

\section{Results}

The test was conducted on a three segment transmission line as shown in Fig. 2. The complete experimental setup is shown in Fig. 8. The setup consists of the power hardware, data acquisition equipment and a computer with the software interface. The test results presented here consisted of running the experiment based on the following user inputs:

1. Structure of the hardware: 3 segment gamma line model and resistive load

2. Parameters to be determined: impedance of reactors, capacitance of capacitors, and load resistance at all frequencies

3. Voltage level(s) to be tested: $120 \mathrm{~V}$ AC

4. Range of frequencies used: $10-500 \mathrm{~Hz}$ at $5 \mathrm{~Hz}$ increments

5. Confidence Level: $99 \%$

6. Number of runs: 100 


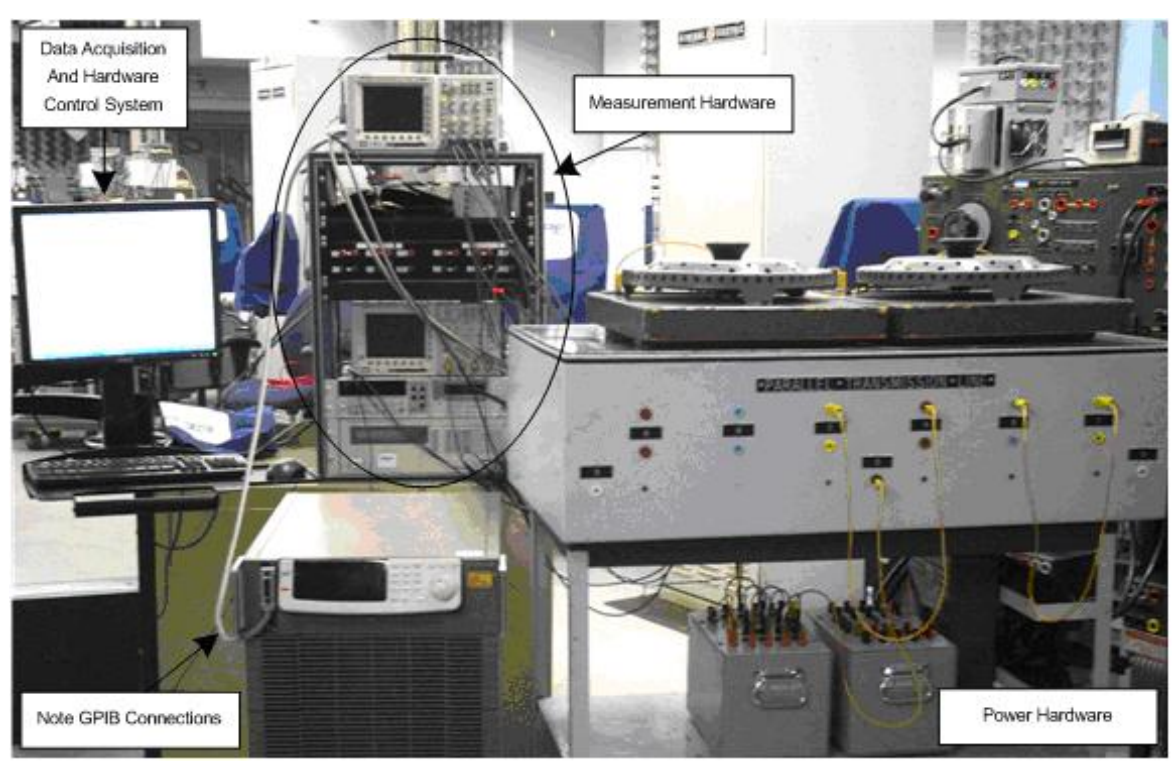

Fig. 8. Complete Test Setup

The frequency range up to $500 \mathrm{~Hz}$ is sufficient for analyzing the $3^{\text {rd }}, 5^{\text {th }}$, and $7^{\text {th }}$ harmonics of a $60 \mathrm{~Hz}$ system which are of interest in many power systems studies and can be present due to line frequency converters and saturation effects from transformers. Results of the test are tabulated in Tables II-V. Table II lists the worst-case standard deviation, interval, and \% from mean for all the measurements across all frequencies tested. Intervals were determined based on the 100 experimental runs, (12), and a confidence level of $99 \%$. All measurements were within the limits specified in this table. Tables III-V list the determined line parameters and their associated intervals at 99\% confidence and various frequencies.

Table II. Statistical Analysis of Measurements

\begin{tabular}{|c|c|c|c|}
\hline & Std Deviation & Interval & $\%$ from mean \\
\hline Voltage Magnitude & $0.222 \mathrm{~V}$ & $\pm 0.056 \mathrm{~V}$ & $0.09 \%$ \\
Current Magnitude & $0.008 \mathrm{~A}$ & $\pm 1.943 \mathrm{~mA}$ & $0.30 \%$ \\
Voltage Phase & $1.877^{\circ}$ & $\pm 0.474^{\circ}$ & $0.85 \%$ \\
Current Phase & $1.825^{\circ}$ & $\pm 0.477^{\circ}$ & $0.77 \%$ \\
\hline
\end{tabular}

The GE reactors are parameterized by a series resistance, $\mathrm{R}$, and a series reactance, $\mathrm{X}$. In other words the impedance of each reactor is $Z=R+j X \Omega$. Generally speaking the reactance for all reactors was determined within a very tight interval. However, the resistance interval was noticeably larger at $99 \%$ confidence. This can be attributed to the sensitivity of the resistance computation to phase angle. Small changes in the measured phase angle resulted in a large change in R. More runs could be conducted to determine the values of $\mathrm{R}$ within a smaller interval. The capacitor values were also determined with a very tight tolerance across most frequencies. The interval was larger at smaller frequencies for the capacitor values.

Fairly good results in transmission line parameter determination have been shown in this example for 100 runs. However, the parameters could be improved further by performing additional runs. In particular, the interval of resistances at higher frequencies could be improved. Automation has been extremely beneficial for this work. It would take weeks to perform 100 runs by manually controlling the source and measurement equipment. More time would also be required for post processing of the results. With this technique these results can be obtained in a matter of hours.

Table III. Line Parameters for Segment 1 at 99\% Confidence 


\begin{tabular}{|c|c|c|c|}
\hline \multirow{2}{*}{ Frequency $(\mathrm{Hz})$} & \multicolumn{3}{|c|}{ Segment 1} \\
\hline & $R(\Omega)$ & $X(\Omega)$ & $C(\mathrm{uF})$ \\
\hline 25 & $0.659+/-0.031$ & $3.562+/-0.008$ & $2.206+/-0.671$ \\
\hline 60 & $0.720+/-0.038$ & $8.522+/-0.010$ & $0.490+/-0.094$ \\
\hline 120 & $0.973+/-0.038$ & $16.976+/-0.019$ & $0.251+/-0.013$ \\
\hline 180 & $1.363+/-0.049$ & $25.351+/-0.027$ & $0.231+/-0.004$ \\
\hline 240 & $1.680+/-0.254$ & $33.570+/-0.144$ & $0.230+/-0.005$ \\
\hline 300 & $2.083+/-0.051$ & $41.813+/-0.047$ & $0.237+/-0.006$ \\
\hline 360 & $2.478+/-0.301$ & $50.052+/-0.122$ & $0.243+/-0.006$ \\
\hline 420 & $3.004+/-0.576$ & $58.031+/-0.216$ & $0.247+/-0.006$ \\
\hline 480 & $3.945+/-0.535$ & $66.035+/-0.202$ & $0.239+/-0.005$ \\
\hline
\end{tabular}


Table IV. Line Parameters for Segment 2 at 99\% Confidence

\begin{tabular}{|c|c|c|c|c|c|c|c|c|c|}
\hline \multirow{2}{*}{ Frequency $(\mathrm{Hz})$} & \multicolumn{9}{|c|}{ Segment 2} \\
\hline & \multicolumn{3}{|c|}{$R(\Omega)$} & \multicolumn{3}{|c|}{$X(\Omega)$} & \multicolumn{3}{|c|}{$C(\mathrm{uF})$} \\
\hline 25 & 0.417 & $+/-$ & 0.038 & 3.574 & $+/-$ & 0.009 & 1.867 & $+/-$ & 0.322 \\
\hline 60 & $0.54 \varsigma$ & $+/-$ & 0.038 & 8.559 & $+/-$ & 0.015 & 0.572 & $+/-$ & 0.060 \\
\hline 120 & 0.686 & $+/-$ & 0.047 & 16.994 & $+/-$ & 0.031 & 0.336 & $+/-$ & 0.016 \\
\hline 180 & 0.903 & $+/-$ & 0.053 & 25.309 & $+/-$ & 0.048 & 0.275 & $+/-$ & 0.007 \\
\hline 240 & $1.17 \varepsilon$ & $+/-$ & 0.197 & 33.577 & $+/-$ & 0.198 & 0.254 & $+/-$ & 0.003 \\
\hline 300 & 1.523 & $+/-$ & 0.076 & 41.725 & $+/-$ & 0.101 & 0.247 & $+/-$ & 0.002 \\
\hline 360 & 1.82 & $+/-$ & 0.267 & 49.853 & $+/-$ & 0.222 & 0.244 & $+1-$ & 0.002 \\
\hline 420 & 2.10 & $+/-$ & 0.547 & 57.797 & $+/-$ & 0.357 & 0.244 & . $+/-$ & 0.003 \\
\hline 480 & 2.908 & $+/-$ & 0.505 & 66.080 & $+/-$ & 0.340 & 0.249 & $+/-$ & 0.003 \\
\hline
\end{tabular}

Table V. Line Parameters for Segment 3 at 99\% Confidence

\begin{tabular}{|c|c|c|c|c|c|c|c|c|c|}
\hline \multirow{2}{*}{ Frequency $(\mathrm{Hz})$} & \multicolumn{9}{|c|}{ Segment 3} \\
\hline & \multicolumn{3}{|c|}{$R(\Omega)$} & \multicolumn{3}{|c|}{$X(\Omega)$} & \multicolumn{3}{|c|}{$C(\mathrm{uF})$} \\
\hline 25 & 1.221 & $+/-$ & 0.033 & 3.603 & $+/-$ & 0.009 & $\overline{0.513}$ & $+/-$ & 0.052 \\
\hline 60 & 1.321 & $+/-$ & 0.034 & 8.619 & $+/-$ & 0.009 & 0.334 & $+/-$ & 0.012 \\
\hline 120 & 1.807 & $+/-$ & 0.042 & 17.191 & $+/-$ & 0.014 & $0.29 s$ & $+/-$ & 0.003 \\
\hline 180 & 2.082 & $+/-$ & 0.052 & 25.673 & $+/-$ & 0.022 & 0.293 & $+/-$ & 0.002 \\
\hline 240 & 2.26 & $+/-$ & 0.098 & 34.184 & $+/-$ & 0.237 & 0.287 & $+/-$ & 0.003 \\
\hline 300 & 2.922 & $+/-$ & 0.061 & 42.502 & $+/-$ & 0.045 & 0.282 & $+/-$ & 0.001 \\
\hline 360 & 3.858 & $+/-$ & 0.143 & 50.788 & $+/-$ & 0.245 & 0.277 & $+/-$ & 0.002 \\
\hline 420 & 4.371 & $+/-$ & 0.249 & 59.349 & $+/-$ & 0.454 & 0.274 & $+/-$ & 0.003 \\
\hline 480 & 5.156 & $+/-$ & 0.290 & 67.450 & $+/-$ & 0.418 & 0.270 & $+/-$ & 0.002 \\
\hline
\end{tabular}

Non-linear loads can also be examined. In this case, FFT analysis can be used to quantify and evaluate terms of equation (3). Tests were performed on the same hardware with a non-linear load to introduce harmonics higher than $500 \mathrm{~Hz}$ to show this functionality. Specifically, the load was a constant DC current source connected to a single phase bridge rectifier. Voltage and current magnitudes/phases of the harmonic components were evaluated using FFT. Results are shown in Figures 9 and 10, normalized to the fundamental magnitude, for voltage and current respectively. Subsequently, resistance and reactance components can be calculated at each frequency using the same approach shown for linear loads.

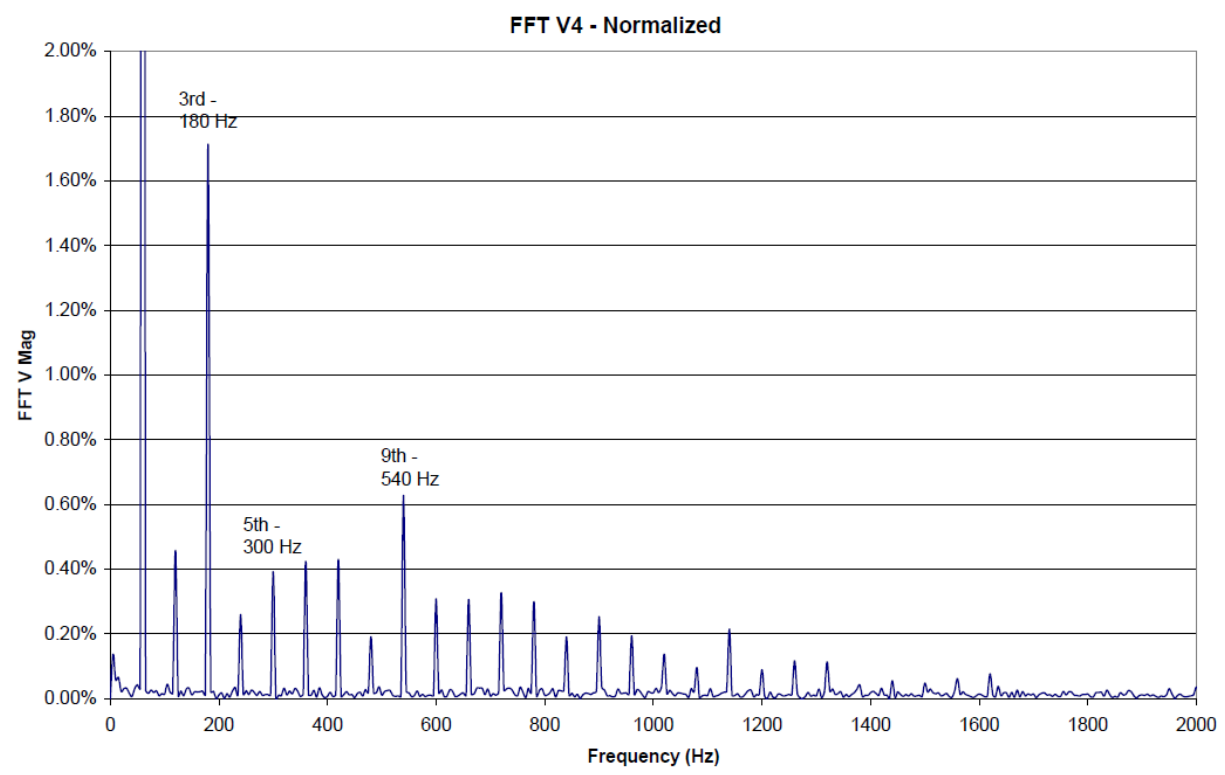

Fig. 9. Load Voltage Magnitude FFT Results for Non-Linear Load 


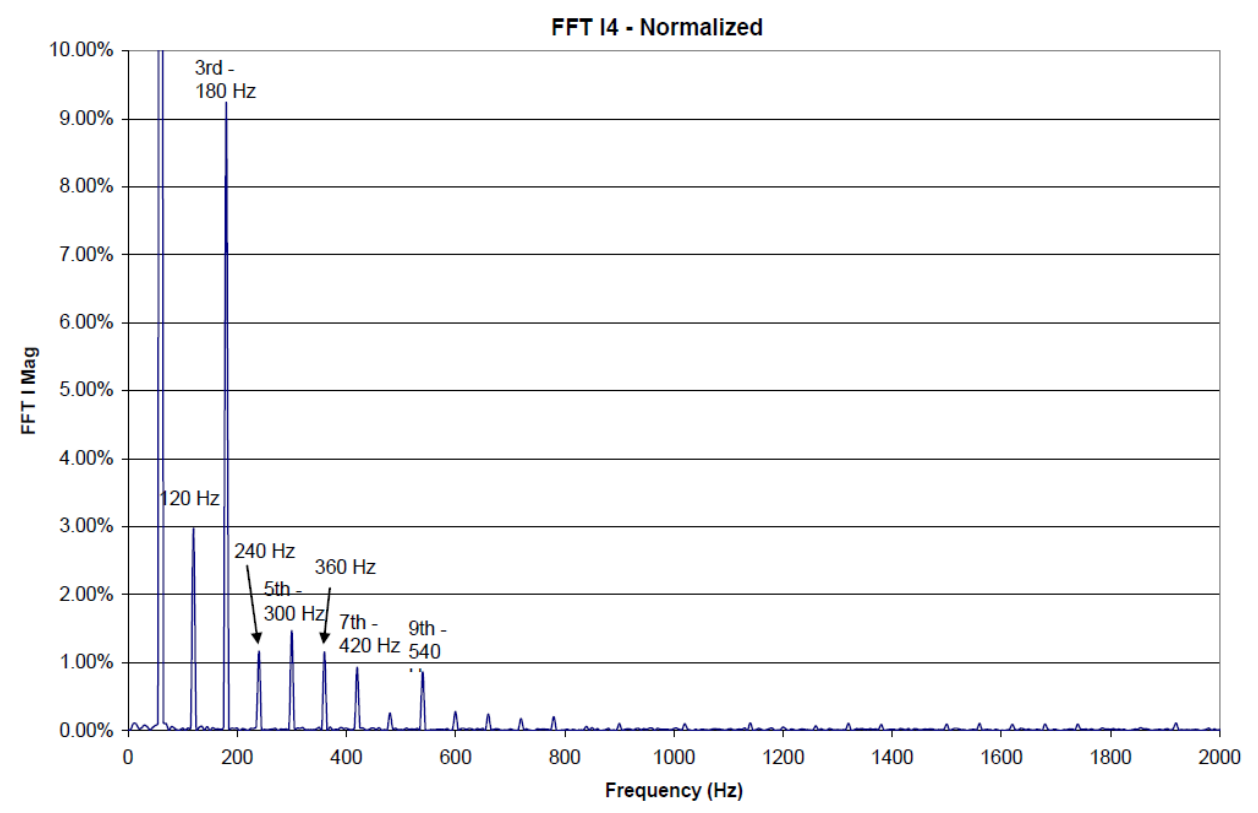

Fig. 10. Load Current Magnitude FFT Results for Non-Linear Load

\section{Conclusion}

An automated method for determining transmission line model parameters at various frequencies has been developed. The power source and measurement system are fully controllable and automated via a custom developed LabVIEW software interface. The data processing incorporates time domain and frequency domain analysis to determine model parameters and utilizes statistical analysis to analyze the quality of the resulting model parameters. The example presented determined the transmission line model hardware parameters accurately within a 99\% confidence level by conducting 100 runs. The main contribution of this work is the development of a systematic process of accurately defining the transmission line parameters and automation of this process. The process presented here is suitable, and may be conducted on, any device that can be parameterized via a current-voltage (I-V) characteristic with appropriate adjustments to transducers, power source, and parameter calculations based on the hardware under test.

\section{Acknowledgment}

This work was supported by ONR N0014-04-1-0404. The views expressed herein are those of the authors and do not reflect the position of the United States Military Academy, the Department of the Army, or the Department of Defense.

\section{References}

[1] G. T. Heydt, Electric Power Quality, 2nd ed.: Stars in a Circle Publications, 1994.

[2] Mohan, Underland, and Robbins, Power Electronics- Converters, Applications and Design, 3rd ed.: John Wiley \& Sons, 2003.

[3] "Voltage Disturbances, Standard EN 50160-Voltage characteristics in public distribution systems," 2004.

[4] V. Cecchi, A. St.Leger, K. Miu, and C. Nwankpa, "Modeling approach for transmisison lines in the presence of nonfundamental frequencies," IEEE Transactions on Power Delivery, vol. 24, pp. 2328-2335, 2009.

[5] P. M. Ramos, F. M. Janeiro, and P. S. Girao, "Uncertainty evaluation of multivariate quantities: A case study on electrical impedance," Measurement vol. 78, pp. 397-411, 2016. 
[6] D. M. Sofian, Z. D. WAng, and S. V. Jayasinghe, "Frequency response analysis in diagnosing transformer winding movements," Proceedings of the 3rd IEE International Conference on Reliability of Transmission and Distribution Networks, pp. 331-335, 2005.

[7] P. M. Ramos, F. M. Janeiro, and T. Radil, "Comparison of impedance measurements in a DSP using ellipse-fit and seven-parameter sine-fit algorithm," Measurement vol. 42, pp. 1370-1379, 2009.

[8] B. Schweighofer, K. M. Raab, and G. Brasseur, "Modeling of high power automotive batteries by the use of an automated test system," IEEE Transactions on Instrumentation and Measurement, vol. 52, pp. 1087-1091, Aug 2003.

[9] A. D. Cheok and N. Ertugrul, "Computer-based automated test measurement system for determining magnetization characteristics of switched reluctance motors," IEEE Transactions on Instrumentation and Measurement, vol. 50, pp. 690-696, Jun 2001.

[10] J. Schoukens, Y. Rolain, G. Simon, and R. Pintelon, "Fully automated spectral analysis of periodic signals," IEEE Transactions on Instrumentation and Measurement, vol. 52, pp. 1021-1024, Aug 2003.

[11] F. Milano, L. Vanfretti, and J. C. Morataya, "An Open Source Power System Virtual Laboratory: The PSAT Case and Experience," IEEE Transactions on Education, vol. 51, pp. 17-23, February 2008.

[12] F. Zouaoui and J. R. Wilson, "Accounting for parameter uncertainty in simulation input modeling," IIE Transactions, vol. 35, pp. 781-792, Sep 2003.

[13] I. Dobson, S. Greene, R. Rajaraman, C. L. DeMarco, F. L. Alvarado, M. Glavic, J. Zhang, and R. Zimmerman, "Electric Power Transfer Capability: Concepts, Applications, Sensitivity and Uncertainty," Power Systems Engineering Research Center PSERC Publication 01-34, November 2001.

[14] S. P. Carullo and C. O. Nwankpa, "Interconnected power systems laboratory: A computer automated instructional facility for power system experiments," IEEE Transactions on Power Systems, vol. 17, pp. 215-222, May 2002.

[15] A. R. Bergen and V. Vittal, Power System Analysis, 2nd ed.: Prentice-Hall, 2000.

[16] ed, Kikusui PCR6000W2. Data Sheet [Online] Available: http://www.kikusui.co.jp/common/product/pdf/pcr-w.pdf.

[17] ed, GE Product Catalog: GEZ-7723D Section12; Page(s): 17-18.

[18] Tektronix, "Digital Phosphor Oscilloscopes: TDS300B Series," 2006 http://www.tek.com.

[19] Tektronix, "Instructions: Tektronix P5200 High Voltage Differential Probe," 1994 http://www.tek.com.

[20] Yokogawa, "User's Manual: 701933 Current Probe," 2004 http://yokogawa.com.

[21] M. Novotny and M. Sedlacek, "RMS value measurement based on classical and modified digital signal processing algorithms," Measurement, vol. 41, pp. 236-250, Apr 2008.

[22] G. E. Mog and E. P. Ribeiro, "One Cycle AC RMS Calculations for Power Quality Monitoring under Frequency Deviation," Proceedings of the 11th International Conference on Harmonics and Quality of Power, pp. 700-705, September 12-15 2004.

[23] P. M. Ramos, M. F. da Silva, and A. C. Serra, "Low frequency impedance measurement using sine-fitting," Measurement, vol. 35, pp. 89-96, Jan 2004.

[24] H. Ku, "Notes on the Use of Propagation of Error Formulas," Research of National Bureau of Standards-C. Engineering and Instrumentation, vol. 70C, pp. 263-273, 1966.

[25] GUM, "Guide to the Expression of Uncertainty in Measurement," ISO, 1995.

[26] B. N. Taylor and C. E. Kuyatt, "Guidelines for Evaluating and Expressing the Uncertainty of NIST Measurement Results," NIST Technical Note 1297, 1994.

[27] A. Papoulis and S. U. Pillai, Probability, Random Variable and Stochastic Processes, 4th ed.: McGraw Hill, 2002.

[28] "LabVIEW 2012," ed: http://www.ni.com/labview/, 2012.

\section{Biographies}

Aaron St. Leger received the B.S., M.S., and Ph.D degrees in Electrical Engineering from Drexel University, Philadelphia, PA, in 2003, 2005 and 2008, respectively. He is currently an Associate Professor in the Electrical Engineering and Computer Science Department, United States Military Academy, West Point, NY.

Valentina Cecchi was born in Rome, Italy. She received her B.S., M.S., and Ph.D. degrees in electrical engineering from Drexel University, Philadelphia, PA in 2005, 2007 and 2010, respectively. She is currently an Assistant Professor in the Department of Electrical and Computer Engineering at the University of North Carolina at Charlotte. 
Megha Basu graduated from Cornell University with a M.S. in electrical Engineering. She is currently a power engineer in the Energy Automation division for Siemens Energy in Minnesota.

Karen Miu (M'98) received the B.S., M.S. and Ph.D. degrees in electrical engineering from Cornell University, Ithaca, NY. She is currently an Associate Professor in the Electrical and Computer Engineering Department, Drexel University, Philadelphia, PA.

Chika O. Nwankpa (M'87) received the Magistr Diploma in electric power systems from Leningrad Polytechnical Institute, USSR, and the Ph.D. degree in the electrical and computer engineering from the Illinois Institute of Technology, Chicago, in 1990. He is currently a Professor of Electrical and Computer Engineering at Drexel University, Philadelphia, PA. 